\title{
Bone marrow mesenchymal stem cell repair of cyclophosphamide-induced ovarian insufficiency in a mouse model
}

This article was published in the following Dove Press journal:

International Journal of Women's Health

15 June 2017

Number of times this article has been viewed

\author{
Ahmed Badawy' \\ Mohamed A Sobh ${ }^{2}$ \\ Mohamed Ahdy ${ }^{3}$ \\ Mohamed Sayed \\ Abdelhafez' \\ 'Department of Obstetrics and \\ Gynecology, ${ }^{2}$ Department of Interna \\ Medicine, ${ }^{3}$ Department of Clinical \\ Pharmacology, Mansoura University, \\ Mansoura, Egypt
}

Objective: Attempting in vivo healing of cyclophosphamide-induced ovarian insufficiency in a mouse model using bone marrow mesenchymal stem cells (BMMSCs).

Methods: Female BALB/c white mice were used to prepare a model for premature ovarian failure by single intraperitoneal injection of cyclophosphamide $(80 \mathrm{mg} / \mathrm{kg})$. Ten mice were injected with BMMSCs and then sacrificed after 21 days for morphometric evaluation of the ovaries. Hormonal profile was evaluated while mice were being sacrificed. Another 10 mice were left for natural breeding with male mice, and 5 of these were injected with BMMSCs. Oocyte-like structures were obtained from 3 mice and were subjected to in vitro fertilization/intracytoplasmic sperm injection.

Results: Morphometric analysis of the ovaries demonstrated the presence of newly formed primordial follicles. Contribution of MSCs to the formation of these follicles was proven by a labeling technique. There was a drop in estradiol and rise in follicle-stimulating hormone levels, followed by resumption of the hormonal levels to near normal 21 days after MSCs therapy. The 5 mice that were injected with MSCs became pregnant after natural breeding. Fertilization and further division was reported in 5 oocytes subjected to intracytoplasmic sperm injection, but division did not continue.

Conclusion: From this proof-of-concept trial, we can say that healing of damaged ovaries after chemotherapy in mice is possible using in vivo therapy with BMMSCs. This should open the gate for a series of animal studies that test the possibility of in vitro maturation of germinal epithelium of the ovary into mature oocytes.

Keywords: cyclophosphamide, stem cell, POF, ovarian insufficiency

\section{Introduction}

A new report by the American Cancer Society ${ }^{1}$ - in collaboration with the National Cancer Institute - estimated that there were almost 14.5 million cancer survivors alive in the USA and that number will grow to almost 19 million by 2024 . The 3 most common cancers among female survivors are breast cancer $(41 \%)$, uterine cancer ( $8 \%$ ), and colorectal cancer $(8 \%)$. Although $46 \%$ of cancer survivors are $\geq 70$ years old, and only $5 \%$ are $<40$ years old, there were almost 50,000 adolescent survivors aged 15-19 years living in the USA. Although the incidence of cancer increases each year, cancer-related mortalities have reduced by almost $1 \%$ per year since the $1990 \mathrm{~s}$. These improved chances for survival have made the long-term effects of therapy more evident. ${ }^{1}$

One of the most demoralizing side effects of cancer treatments is destruction of the reproductive system, which in young girls and women $<40$ years old is frequently associated with premature ovarian failure (POF) and infertility. ${ }^{2,3}$ These effects are
Correspondence: Ahmed Badawy Department of Obstetrics and Gynecology, Mansoura University Hospital, Elgomhouria Street, Mansoura City 35I II, Dakahlia, Egypt

Tel +20 502946830

Email ambadawy@hotmail.com
International Journal of Women's Health 20I7:9 44I-447

$44 I$ 
the result of cytotoxic effects on the oocyte-granulosa cell complex. ${ }^{4,5}$ Pathologic destruction of oocytes was thought to be irreversible due to the well-known fact that oocytes are a fixed and non-renewing cohort at birth. ${ }^{6,7}$ Despite the revolutionary policies of fertility preservation adopted for implementation against the oocyte-destructive cancer therapy, there is still a considerable cohort of women who have lost a number of oocytes. ${ }^{8}$

There are promising reports about the possibility of unexpected return of ovarian function and fertility in patients undergoing bone marrow (BM) transplantations. ${ }^{9-11}$ One conceivable explanation for this may be due to the healing power of mesenchymal stem cells (MSCs). There are many controversial reports about this issue, and scientists are still skeptical about the concept of neo-oogenesis brought about by the mesenchymal or ovarian stem cells. ${ }^{12-14}$

In this proof-of-concept study, we attempt to treat cyclophosphamide (CPA)-induced ovarian insufficiency in vivo, in a mouse model, using bone marrow mesenchymal stem cells (BMMSCs).

\section{Materials and methods}

\section{Animals}

Female BALB/c white mice were obtained from the animal house of Mansoura Experimental Research Center, Mansoura University, Egypt. The mice were aged 4-6 weeks. The mice were kept on a $12 \mathrm{~h} / 12 \mathrm{~h}$ light/dark cycle and were provided with food and water ad libitum. All mice were given a single intraperitoneal injection of CPA at a dose of $80 \mathrm{mg} / \mathrm{kg}$ body weight (Baxter Healthcare Corporation, Deerfield, IL, USA), with the drug being dissolved in $0.9 \%$ saline. As demonstrated before, the model is ready for use between 4-28 days of CPA injection, the time of complete disappearance of primordial follicles.

The Institutional Review Board of Mansoura Faculty of Medicine approved the procedures and research as per the principle contained in Clause 3.1.1 of the Australian Code of Practice for the Care and Use of Animals for Scientific Purposes.

\section{Establishment of BMMSCs}

The BM was obtained from the femur and tibia of the 7- to 8-week-old BALB/c white mice. For each experiment, BM cells were isolated from 4 donor mice. The bones were sterilized by immersion in $70 \%$ ethanol. The ends of the bones were cut and BM was extruded by inserting a needle at one end through the bone shaft and injecting tissue culture media (Dulbecco's Modified Eagle's Medium; Sigma Chemical Comp., St Louis, MO, USA) containing 10\% fetal bovine serum (FBS, Sigma Chemicals, Perth, Australia). The effluent was collected in sterile tubes. Gentle pipetting resulted in generation of a single-cell suspension. The BM cells were counted and plated with a concentration of $10 \times 10^{6} / \mathrm{mL}$ in T-75 flasks. The cells were then cultured in Dulbecco's Modified Eagle's Medium containing 10\% heat-inactivated FBS, penicillin $(100 \mathrm{U} / \mathrm{mL})$, and streptomycin $(100 \mu \mathrm{g} / \mathrm{mL})$ and incubated at $37^{\circ} \mathrm{C}$ in a humidified atmosphere that contained $5 \% \mathrm{CO}_{2}$. Media were changed after the first 4 days and every 3 days thereafter. Nonadherent hematopoietic cells were removed when media were changed. After a mean of 21 days, cells reached subconfluence and were detached with trypsin/EDTA, reseeded at $4 \times 10^{3}$ cells $/ \mathrm{cm}^{2}$, and used for experiments after the third passage. The MSC features were demonstrated by the cells possessing the typical spindleshaped morphology.

\section{Therapy using MSCs followed by morphometric evaluation of the ovaries and clarification of homing of MSCs in the ovaries}

Ten mice with POF were used in this stage. Five mice were injected through the tail vein with $0.5 \times 10^{6}$ of MSCs supplemented in free media. Injection was done on day 4 after CPA injection, the time of complete disappearance of the primordial follicles from the ovary. The MSCs were labeled with iron oxide (Sigma Chemical) and injected through the tail vein of another 5 mice with POF.

The injected animals were sacrificed 21 days after MSCs injection and the ovaries were collected in formalin or normal saline. In the first 5 injected animals, routine hematoxylin and eosin staining of 4-5 $\mu \mathrm{m}$ sections was done to evaluate the morphologic changes of the ovary after MSCs injection. The number of atretic primordial, primary, and preantral follicles was then determined. Immature follicles were scored as atretic if the oocytes were degenerating (convoluted, condensed) or fragmented. Grossly atretic immature follicles lacking oocyte remnants were not included in the analyses. In the 5 animals injected with labeled MSCs, testing for the homing of MSCs in the ovaries was done by staining of the ovarian sections with Prussian blue for iron oxide labeling of the newly formed oocytes.

\section{Biochemical evaluation of therapy}

Serum hormonal profile of the treated mice, including estradiol (E2) and follicle-stimulating hormone (FSH), was evaluated. A blood sample $(1 \mathrm{~mL})$ was collected from each mouse while it was being sacrificed (on day 21 after BMMSCs injection). The collected sample was left to clot, centrifuged, 
and the serum then separated and frozen for assay of E2 and FSH levels. Serum E2 was measured by radioimmunoassay using a commercial, direct, double-antibody kit from Pantex (Santa Monica, CA, USA). Serum FSH was measured using Mouse FSH ELISA Kit (Competitive EIA) from LifeSpan BioSciences (Seattle, WA, USA).

\section{Attempt at natural breeding}

Another set of POF mice, containing a group of 10 mice, was divided into 2 groups, 5 mice in each group. One group (5 mice) was injected with MSCs (for healing of the ovarian damage) and was left for natural breeding with male mice, while the other group (5 mice) was not injected with MSCs and also left for natural breeding. Adult males of proven fertility were housed with females at a ratio of $1 / 2$. Males were randomly rotated among cages after each pregnancy.

\section{In vitro fertilization (IVF)/intracytoplasmic sperm injection (ICSI) for newly formed oocytes}

The oocyte-like structures, obtained from the ovaries of the POF model mice on day 21 after being treated with MSCs, were incubated or injected with isolated mouse sperm in vitro to obtain fertilized oocytes by the technique of IVF/ICSI.

\section{Obtaining the in vivo new oocytes}

POF mice (3 mice) were injected with MSCs (for healing of the ovarian damage). Animals were euthanized by inhalation of isoflurane in oxygen. The surgical site was cleaned with $70 \%$ alcohol, followed by povidone iodine solution, then the oviducts were removed, and the cumulus oocyte masses from the ampullae were dissected. Surgery was performed by using a stereomicroscope. Oocytes were subjected to in vitro maturation (IVM) in culture medium for 24 hours before being used for the next steps.

\section{Obtaining the mice sperm}

Three mature male BALB/c white mice were sacrificed and their testicles obtained by meticulous dissection. The testicles were then divided into halves using a sharp knife and incubated for 2 hours in Mouse Vitro Fert medium (MVF; Cook Medical, Brisbane, QLD, Australia) to allow the sperm to move by swim-up technique into the supernatant fluid layer; these sperm were then collected by using a pipette into the MVF medium and were ready for use in IVF/ICSI.

\section{New oocytes undergoing IVF/ICSI}

The obtained mature oocytes were divided into 2 groups for IVF and ICSI. Cumulus oocyte masses were collected into a culture dish containing phosphate-buffered saline with $4 \mathrm{mg} / \mathrm{mL}$ bovine serum albumin. Cumulus cells were dissociated from the oocytes with hyaluronidase $(0.3 \mathrm{mg} / \mathrm{mL})$. The cumulus cells were not completely removed. The numbers of live, dead, and fragmented oocytes collected from the left and right oviduct of each mouse were counted. IVF/ICSI was performed according to the standard technique, and the MVF medium was used as the fertilization and sperm collection medium.

\section{Assessment of fertilization}

About 16-18 hours after incubation or microinjection, oocytes were checked for the presence of pronuclei and polar bodies. After another 24 hours, rechecking of the division and scoring of the embryos (eg, equal size of blastomeres) was done under the stereomicroscope. Embryo culture continued to reach blastocyst stage.

\section{Results}

As demonstrated in our POF model, the BALB/c white mice were most sensitive to primordial follicle destruction by CPA at a dose of $80 \mathrm{mg} / \mathrm{kg}$ between 72 and 96 hours after injection (nearly 100\%), and this effect continued till at least 4 weeks. We decided to use the model between day 4 till day 28 after injection for further phases of the trial.

\section{Results of morphometric evaluation}

After establishment of BMMSCs and injecting $0.5 \times 10^{6}$ cells in the tail vein of each mouse in the POF model mice (10 mice), we waited 21 days and then sacrificed these mice to obtain their ovaries. Morphometric analysis of the ovaries demonstrated the presence of newly formed primordial follicles (Figure 1). To confirm the contribution of the MSCs to the formation of the new primordial follicles, we used the labeling technique. The BMMSCs were labeled with iron oxide (a substance which does not exist in the ovary). Prussian blue stain was observed in the newly formed follicles, proving that the MSCs were contributing either partially or completely to the formation or healing of the primordial follicles in the ovaries (Figure 2).

\section{Results of biochemical evaluation of therapy}

This work demonstrated clearly the drop in E2 level and rise in FSH in the POF mouse model followed by resumption of the hormonal levels to near normal after 21 days of MSCs therapy (Table 1). 


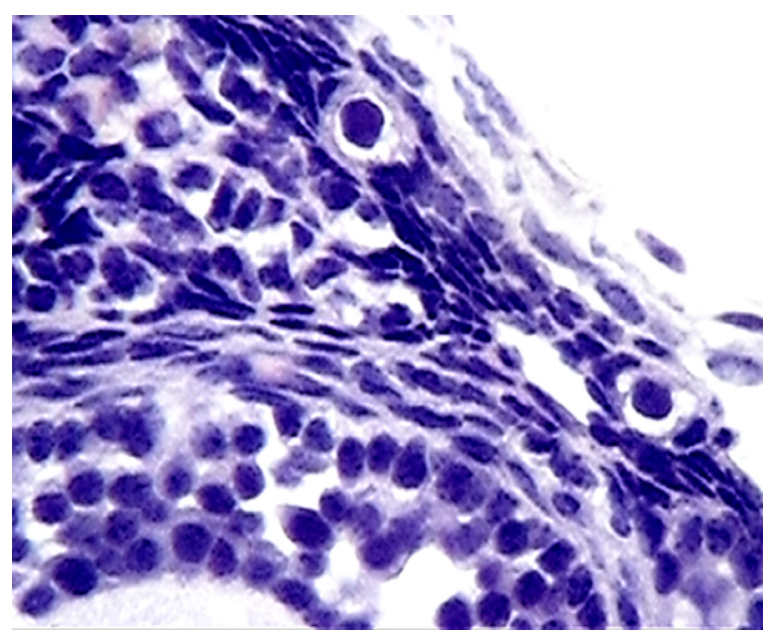

Figure I New primordial oocytes formed in the POF ovaries 21 days after MSCs injection (H\&E stain).

Abbreviations: H\&E, hematoxylin and eosin; MSCs, mesenchymal stem cells; POF, premature ovarian failure.

\section{Results of natural breeding}

In the POF mice (10 mice) which were left for natural breeding with adult male mice with proven fertility, only the 5 mice that were injected with MSCs became pregnant while the other 5 mice that were not injected with MSCs remained non-pregnant after 4 weeks of observation (Figures 3 and 4). This proved that mice treated with MSCs regained their fertility.

\section{Results of IVF/ICSI of new oocytes}

In this step, 9 oocytes were isolated (Figure 5) and 4 oocytes were separated and incubated with sperm (as described

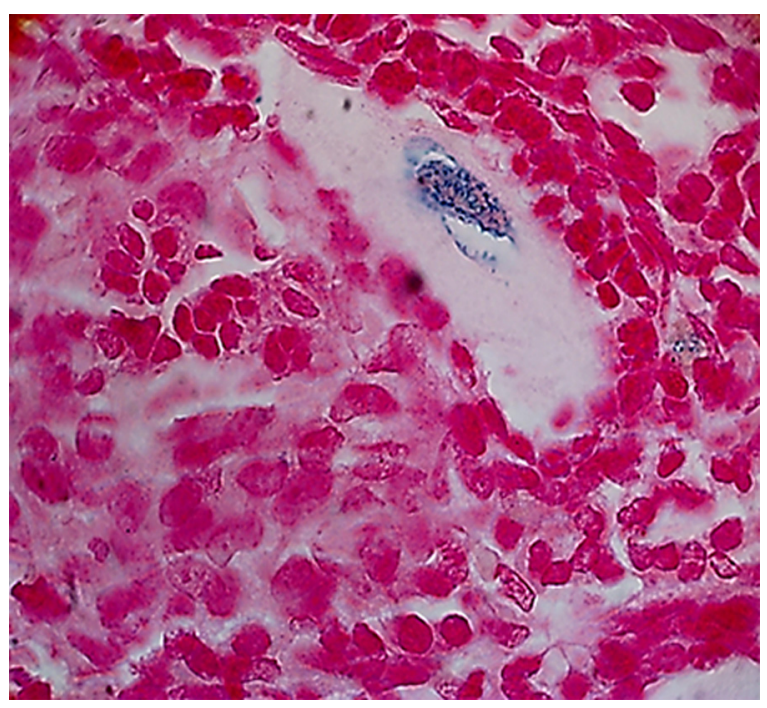

Figure 2 New primordial oocytes formed in the POF ovaries 21 days after MSCs injection with stem cells labeled with iron oxide (Prussian blue stain).

Abbreviations: MSCs, mesenchymal stem cells; POF, premature ovarian failure.
Table I Hormonal profile on day 2 I after BMMSCs therapy

\begin{tabular}{llll}
\hline Hormone & $\begin{array}{l}\text { Treated POF } \\
\text { model }(\mathbf{n}=\mathbf{5})\end{array}$ & $\begin{array}{l}\text { Non-treated } \\
\text { POF model }(\mathbf{n}=\mathbf{5})\end{array}$ & P-value \\
\hline E2 level $(\mathrm{pg} / \mathrm{mL})$ & $249.41 \pm 6.91$ & $161.22 \pm 9.11$ & $<0.001$ \\
FSH level $(\mathrm{ng} / \mathrm{mL})$ & $0.34 \pm 0.07$ & $0.59 \pm 0.06$ & $<0.001$ \\
\hline
\end{tabular}

Note: Data are presented as mean \pm standard deviation.

Abbreviations: BMMSCs, bone marrow mesenchymal stem cells; E2, estradiol; $\mathrm{FSH}$, follicle-stimulating hormone; POF, premature ovarian failure.

earlier) for IVF. Five oocytes were denuded and injected as described before (Figure 6). No fertilization was detected in the IVF group, but fertilization and further division was reported in the ICSI group of oocytes; however, division at this stage of the study did not continue (Figures 7 and 8).

\section{Discussion}

It seems that BMMSCs can contribute partially or completely to the formation of primordial follicles in the ovaries of $\mathrm{BALB} / \mathrm{c}$ white mice with CPA-induced ovarian failure. This study is one of proof-of-concept trials from different centers trying to answer the eternal question of whether there is neo-oogenesis or not. ${ }^{11}$

In this study, we used the pre-prepared model of BALB/c white mice injected with $80 \mathrm{mg} / \mathrm{kg}$ of CPA to induce a state of POF. Our previous study (unpublished data, Badawy et al) proved that between 4 days and 4 weeks of CPA injection, the ovary becomes nearly devoid of primordial follicles. Any attempt of treatment using MSCs was done during this period, ie, from day 4 to day 28. The BMMSCs used for therapy of the POF model were characterized by typical spindle-shaped morphology after a mean of 21 days. The third passage of culture when cells reached subconfluence and were detached with trypsin/EDTA and reseeded at $4 \times 10^{3}$ cells $/ \mathrm{cm}^{2}$.

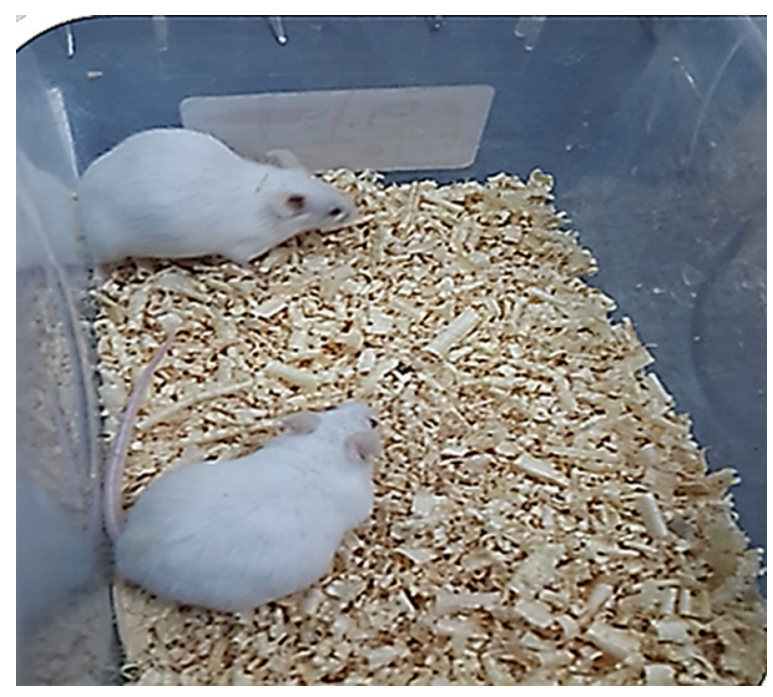

Figure 3 Healed mice in an advanced stage of pregnancy. 


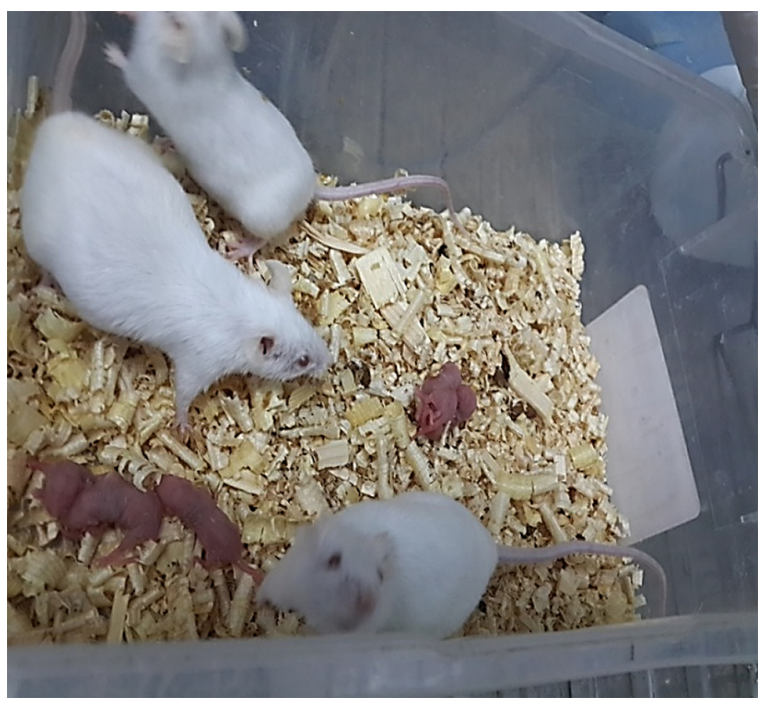

Figure 4 Healed mice after delivery.

In the first part of this study, there were evident abundant primordial follicles in the ovaries 21 days after the MSCs injection in the tail vein of the animals. According to our POF model, there should be no primordial follicles during this period, and so we considered this to be the first proof of the possibility of newly formed or repaired primordial follicles. However, it can be argued that these primordial follicles represent the $1 \%-2 \%$ of follicles that were not destroyed by CPA injection. Nevertheless, the numbers present in the sections prepared from the ovarian sections were more than what can be considered as having survived the destruction by CPA.

In the second part of the study, MSCs used for therapy were labeled with iron oxide before use in the trial. ${ }^{15}$ Iron

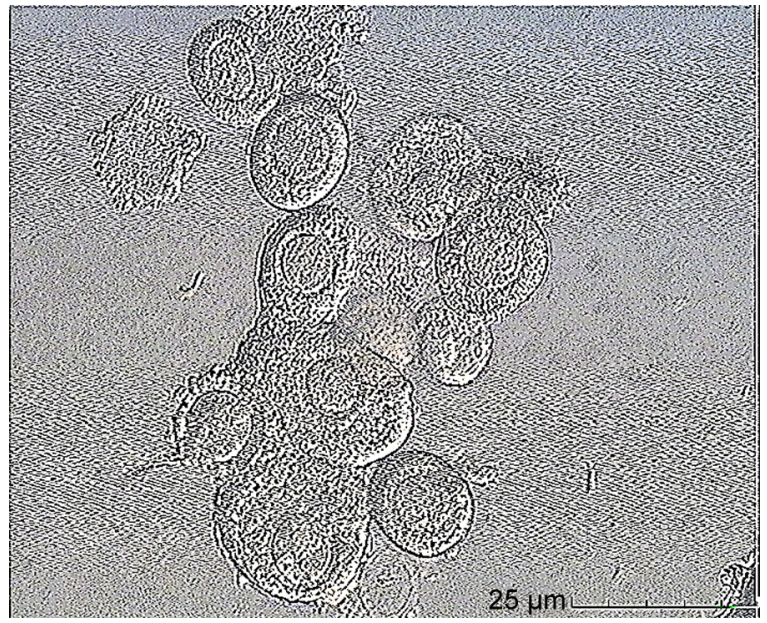

Figure 5 Oocytes collected from the mouse ovaries under phase contrast microscope.

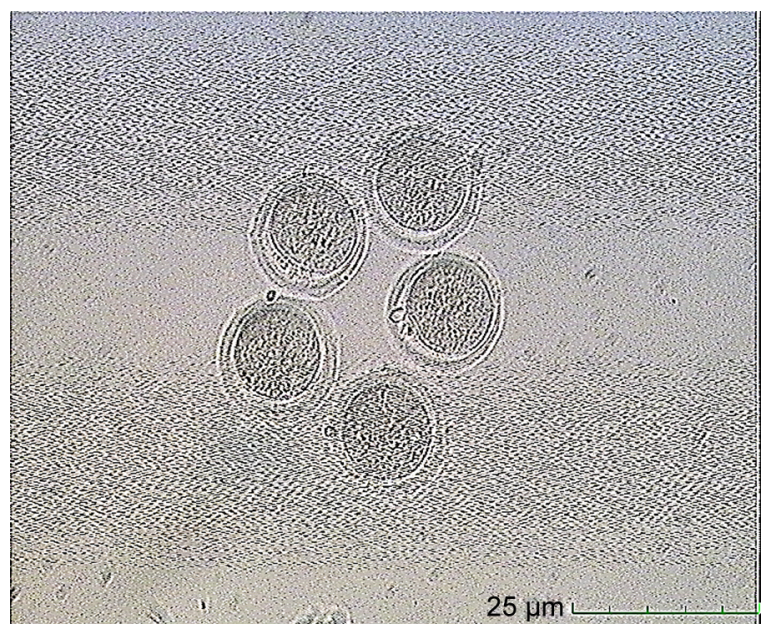

Figure 6 Oocytes collected from the mice ovaries after denudation for ICSI (under phase contrast microscope).

Abbreviation: ICSI, intracytoplasmic sperm injection.

oxide is generally not present in normal ovarian cells. The presence of iron oxide inside the primordial follicles in the ovarian section was the second proof of the partial or complete effect of the injected stem cells in the repair or formation of the primordial follicles. The resumption of ovarian function was associated with resumption of the normal serum hormonal levels (E2 and FSH) (Table 1).

In the third part of the trial, we were aiming to prove the viability of the newly formed oocytes by natural breeding. We housed the treated female mice with fertile male white mice that were rotated among the untreated mice. The healed female mice became pregnant and delivered healthy newborn mice, while the untreated female mice remained infertile. This was the third proof of the value of the injected

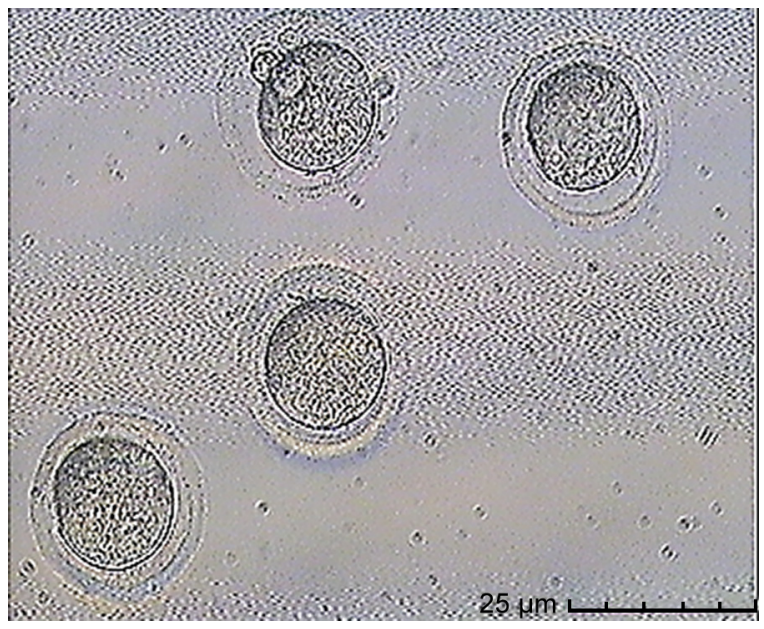

Figure 7 Oocytes collected from the mice ovaries after fertilization with cytoplasmic regression apparent (under phase contrast microscope). 


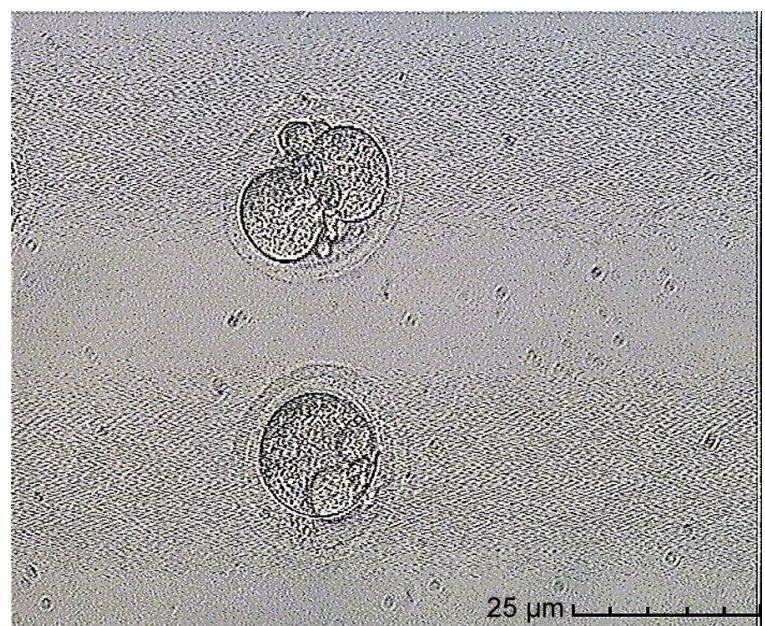

Figure 8 Fertilized oocytes collected from the mice ovaries after 48 hours; 2 cell stages (under phase contrast microscope).

BMMSCs in restoring the normal ovarian function as regards the ovulation or hormonal production.

We decided to use the technology of IVF/ICSI to prove the concept of successful healing of the primordial follicles and restoration of the fertility of the ovary after being treated with chemotherapeutic agents with multiple side effects. We managed to extract the primordial follicles, not the mature follicles, from the ovary and subject them to IVM, to be then used for IVF/ICSI. We observed that the extracted oocytes were morphologically normal and matured when subjected to IVM technique. The oocyte cumulus complex was morphologically normal, which is in agreement with what was demonstrated by many researchers previously, ie, CPA affects the oocytes more than the granulosa cells., ${ }^{4,5}$ Oocytes mixed with mice sperm and left for IVF did not show evidence of fertilization despite the favorable environment. This is not an uncommon finding, especially after IVM, when we extrapolate data from the human studies. On the other hand, the oocytes subjected to ICSI using mice sperm demonstrated signs of fertilization and division into the 2 cell stage but not beyond. The results of this part of the study proved that the obtained oocytes after MSCs therapy were viable, healthy, and a candidate for fertilization. This part of the study was a simulation for what will be done in the future human trials in an attempt to treat the infertility that follows chemotherapyinduced ovarian insufficiency.

We are aware of the shortcomings of the study, particularly in the proper characterization of the SCs before and after injection into the animal model using gene expression and immunohistochemical stains. However, this is still a proof-of-concept trial which should be repeated in a more tightly controlled way.

\section{Conclusion}

From this proof-of-concept trial, we can say that healing of damaged ovaries after chemotherapy in mice is possible following in vivo therapy with BMMSCs. This should open the gate for a series of animal studies that test the possibility of IVM of germinal epithelium of the ovary into mature oocytes.

\section{Acknowledgment}

This project was supported financially by the Science and Technology Development Fund (STDF), Egypt, Grant No 4223.

\section{Disclosure}

The authors report no conflicts of interest in this work.

\section{References}

1. American Cancer Society. Cancer Facts and Figures 2015. Atlanta: American Cancer Society; 2015.

2. Meirow D, Nugent D. The effects of radiotherapy and chemotherapy on female reproduction. Hum Reprod Update. 2001;7(6):535-543.

3. Wenzel L, Dogan-Ates A, Habbal R, et al. Defining and measuring reproductive concerns of female cancer survivors. J Natl Cancer Inst Monogr. 2005;(34):94-98.

4. Gulyas BJ, Mattison DR. Degeneration of mouse oocytes in response to polycyclic aromatic hydrocarbons. Anat Rec. 1979;193(4):863-882.

5. Byskov AG. Follicular atresia. In: Jones RE, editor. The Vertebrate Ovary. New York, NY: Plenum Press; 1987:533-562.

6. Zuckerman S. The number of oocytes in the mature ovary. Recent Prog Hormone Res. 1951;6:63-109.

7. Zuckerman S, Baker T. The development of the ovary and the process of oogenesis. Ovary. 1977;1:41-67.

8. Lee SJ, Schover LR, Partridge AH, et al. American Society of Clinical Oncology recommendations on fertility preservation in cancer patients. J Clin Oncol. 2006;24(18):2917-2931.

9. Salooja N, Szydlo RM, Socie G, et al. Pregnancy outcomes after peripheral blood or bone marrow transplantation: a retrospective survey. Lancet. 2001;358(9278):271-276.

10. Hershlag A, Schuster MW. Return of fertility after autologous stem cell transplantation. Fertil Steril. 2002;77(2):419-421.

11. Johnson J, Canning J, Kaneko T, Pru JK, Tilly JL. Germline stem cells and follicular renewal in the postnatal mammalian ovary. Nature. 2004;428(6979):145-150.

12. Tilly JL. Commuting the death sentence: how oocytes strive to survive. Nat Rev Mol Cell Biol. 2001;2(11):838-848.

13. Byskov AG, Faddy MJ, Lemmen JG, Andersen CY. Eggs forever? Differentiation. 2005;73(9-10):438-446.

14. Telfer EE, Gosden RG, Byskov AG, et al. On regenerating the ovary and generating controversy. Cell. 2005;122(6):821-822.

15. Hanot CC, Choi YS, Anani TB, Soundarrajan D, David AE. Effects of iron-oxide nanoparticle surface chemistry on uptake kinetics and cytotoxicity in CHO-K1 cells. Int J Mol Sci. 2015;17(1). 
International Journal of Women's Health

Dovepress

\section{Publish your work in this journal}

The International Journal of Women's Health is an international, peerreviewed open-access journal publishing original research, reports, editorials, reviews and commentaries on all aspects of women's healthcare including gynecology, obstetrics, and breast cancer. The manuscript management system is completely online and includes

Submit your manuscript here: http://www.dovepress.com/international-journal-of-womens-health-journa a very quick and fair peer-review system, which is all easy to use. Visit http://www.dovepress.com/testimonials.php to read real quotes from published authors. 